\title{
Joint Workshop
}

\section{J-1}

Live Cell Fluorescence Imaging with the Leica SR GSD System. URS KUEDERLI. W. Nuhsbaum Inc. Indianapolis, IN 46250. Email: kuederli@nuhsbaum.com

Microscopy has developed into one of the most important tools in live science research. However, a resolution gap exists between light and electron microscopy with respect to detailed cellular structures that even hi resolution confocal microscopy can't resolve. Working with Stefan Hell of the Max Plank Institute Leica has developed a ground state depletion microscope platform, the Leica SR GSD. This system allows the user to generate and analyze images with the highest lateral resolution down to $20 \mathrm{nM}$. Such resolution enables substructure analysis of specimens such as viruses, endosomes, and exosomes impacting neurobiology, cell biology, virology, microbiology, and physiology. This platform is emerging as a superb tool for imaging structures that cannot be seen with current diffraction limited light microscopes and confocal microscopy.

\section{J-2}

Automated Fluorescence Microscopy Demystified. C. J. FRESON. Life Technologies, Carlsbad, CA. Email: chris.freson@lifetch.com

iPads, iPhones, Androids and Windows tablets are becoming a part of the fabric of our lives. They simplify routine tasks in our daily lives, and lower barriers to accomplish things that at one time in the very recent past would have been considered extremely complex - and prohibitively expensive. People no longer need to spend the kind if time it once took to develop proficiencies that in the past simply allowed them to generate the data required to address their fundamental questions. This talk will discuss how these philosophies are being applied to microscopy in today's interdisciplinary research environment. 